\section{Secondary findings on virtual panels: opportunities, challenges, and potential for preventive medicine}

We read with interest the commentary by Dr. Biesecker entitled "Secondary findings in exome slices, virtual panels and anticipatory sequencing," proposing the demarcation and return of secondary findings as outlined by the American College of Medical Genetics and Genomics (ACMG), identified through multigene panels, exome slices, and anticipatory sequencing. We agree that this is a key issue for the ACMG to address and wish to contribute to the discussion of this important topic.

In the virtual panels section, Dr. Biesecker succinctly describes the potential for return of secondary findings across clinical areas (ACMG59 cancer and cardiovascular findings when an epilepsy panel is ordered), when the data is generated with a common molecular process/platform.

Does a variant in an unrequisitioned gene represent a secondary finding if the gene belongs to the clinical area for which the testing was initially indicated? Specifically, healthcare professionals (HCPs) often order a narrow panel for patients based on clinical indication. For example, in a patient with personal or family history of breast cancer, an HCP may order BRCA1 and BRCA2 only, or an 11-gene guidelinesbased breast cancer panel (e.g., ATM, BRCA1, BRCA2, CDH1, CHEK2, NBN, NF1, PALB2, PTEN, STK11, and TP53). At the same time, data may be generated for some or all of the hereditary cancer syndrome (HCS) genes on the ACMG59, including such genes as MLH1, MSH2, MSH6, PMS2, EPCAM, APC, MEN1, and RET, among others. On the one hand, one might argue that variants in these other HCS genes are not secondary findings because other providers might have ordered them for the same indication because they prefer broader panels. On the other hand, the same would not be true for patients undergoing clinically indicated genetic testing for a cardiovascular condition, such as familial hypercholesterolemia (FH). Here, an HCP may order an FH-focused panel (e.g., LDLR, APOB, PCSK9, and LDLRAP1). At the same time, data may be generated for some or all of the cardiovascular genes on the ACMG59, including such genes as FBN1, TGFBR1, TGFBR2, MYH7, RYR2, PKP2, KCNQ1, and $S C N 5 A$, among others, that would not ordinarily be examined in a patient with a dyslipidemia. Although it is not always obvious what constitutes an expanded panel and what is a true secondary finding, these examples represent additional opportunities for patients to obtain information valuable to their health in the ACMG59 genes.

It could be suggested that secondary findings should not be offered in conjunction with multigene panels/exome slices because the physicians ordering the panels are doing so for a specific clinical indication and are not interested in, or do not want the possibility of, secondary findings. However, the same was said of physicians ordering exome sequencing or genome sequencing (ES/GS) at the time of release of the original ACMG56 guidelines. In fact, studies conducted at the time of the original ACMG56 guidelines release showed that there were both primary care clinicians as well as genetics professionals who opposed even the offering of actionable incidental findings to adult patients undergoing ES/GS, ${ }^{2}$ let alone the mandatory release of secondary findings as originally recommended by the ACMG. One of the reasons for this opposition could have been related to the potential that "[a]n IF [incidental finding] may fall outside the expertise of the clinician who ordered the test, and referral to others may be necessary." ${ }^{3}$ However, despite this clinician reluctance, the ACMG proceeded with release of the 2013 ACMG56 recommendations, which have, over time, gained broad acceptance. Similarly, we would advocate that the ACMG consider recommending the option of secondary findings in conjunction with multigene panels/exome slices/ anticipatory sequencing despite potential physician apprehension related to implementation.

Finally, Dr. Biesecker raises the hypothetical construct in which a patient has suffered harm due to the "...lack of an opportunity to receive secondary findings." This statement suggests that the offering of secondary findings represents a potential benefit, in the form of personalized preventive medicine, to patients who engage in this opportunity, with which we would agree. In fact, the impact of this benefit population-wide would be significantly increased if secondary findings were offered from multigene panels/exome slices/ anticipatory sequencing. Let's consider a hypothetical case where in addition to ES, secondary findings are offered from multigene panels and exome slices classified as panels. According to insurance claim clearinghouse data, recent estimates indicate that clinical ES (as represented by Current Procedural Terminology [CPT] codes 81415,81416 ) is ordered annually nationwide for over 1600 patients (this could represent up to 4800 patients if all were trios). The same insurance claim clearinghouse data indicate that the number of clinical multigene panels/exome slices ordered for hereditary breast and ovarian cancer (HBOC) and hereditary colon cancer (CRC) alone (as represented by CPT codes 81162 , 81211 and codes 81435,81436 , respectively) is over 90,000 annually. This suggests the potential for a 50 -fold increased magnitude of additional positive impact to patients' preventive health care, through opportunistic screening of individuals, if 
return of secondary findings from virtual panels/exome slices were implemented for HBOC and CRC diagnostic panels alone. At the same time, this suggests that any conversation around expansion of the ACMG secondary findings guidelines to include multigene panels/exome slices/anticipatory sequencing needs to consider the implementation burden this would create. This would certainly require novel genetics service models for pretest counseling, delivery of the resulting volume of actionable secondary findings, and coordination of appropriate specialty care follow up where indicated, issues with which the ACMG is already actively engaged.

Accordingly, we support and will continue to abide by the current ACMG guidelines regarding return of secondary findings for ES/GS. At the same time, we join Dr. Biesecker's call for the ACMG to consider creating secondary findings recommendations explicitly covering multigene panels/exome slices/anticipatory sequencing. Such recommendations, consistent with the spirit of the original guidance, would expand access to secondary findings and the associated precision preventive health-care benefits for patients and their family members.

\section{ACKNOWLEDGEMENTS}

We appreciate the assistance of John Moore in retrieving the described insurance clearinghouse claims data.

\section{CONFLICT OF INTEREST}

By way of disclosure, all authors are employees of and are shareholders in Invitae Corp. Invitae is a commercial genetic testing laboratory that offers genetic testing for hereditary conditions, including multigene panels and clinical exomes, areas of relevance for this paper.

Edward D. Esplin, $M D, P h D^{1}$, Eden Haverfield, $P h D^{1}$, Shan Yang, $P h D^{1}$, Swaroop Aradhya, $P h D^{1}$ and

Robert L. Nussbaum, $M D^{1}$

${ }^{1}$ Invitae, San Francisco, CA, USA. Correspondence: Edward D. Esplin (ed. esplin@invitae.com)

\section{REFERENCES}

1. Biesecker LG. Secondary findings in exome slices, virtual panels, and anticipatory sequencing. Genet Med. 2018 May 22; https://doi.org/ 10.1038/s41436-018-0019-3 [Epub ahead of print].

2. Mackley MP, Fletcher $B$, Parker $M$, Watkins $H$, Ormondroyd $E$. Stakeholder views on secondary findings in whole-genome and wholeexome sequencing: a systematic review of quantitative and qualitative studies. Genet Med. 2017;19:283-293.

3. Crawford G, Foulds N, Fenwick A, Hallowell N, Lucassen A. Genetic medicine and incidental findings: it is more complicated than deciding whether to disclose or not. Genet Med. 2013;15:896-899.

Advance online publication 24 September 2018. doi:10.1038/s41436-018-0302-3 